\title{
DETERMINAÇÃO DOS VALORES DE COLESTEROL-LDL DE EQUINOS POR ESPECTROFOTOMETRIA SUBMETIDOS A EXERCÍCIO EM ESTEIRA E SUPLEMENTADOS COMVITAMINA "E"
}

\author{
Pollyanny Ferreira Costa ${ }^{1}$ \\ Maria Carolina Gobbi de Oliveira ${ }^{1}$ \\ Letícia Andreza Yonezawa ${ }^{2}$ \\ Marcos Jun Watanabe ${ }^{3}$ \\ Aguemi Kohayagawa ${ }^{4}$ \\ Tatiana de Sousa Barbosa ${ }^{5}$
}

\begin{abstract}
RESUMO
A lipoproteína de baixa densidade (LDL) faz parte de um grupo de lipoproteínas que, por sua natureza anfifílica, são responsáveis pelo transporte de colesterol plasmático, sendo esse carreamento importante, uma vez que o colesterol é insolúvel no plasma sanguíneo. Sabe-se que a principal fonte de energia dos cavalos, para a contração basal dos músculos, é obtida a partir dos ácidos graxos livres proveniente do tecido adiposo e os corpos cetônicos, produtos da quebra de ácidos graxos provenientes do fígado, processo que gera espécies reativas de oxigênio (ERO), que, em excesso, leva a um estresse oxidativo celular. Para evitar o dano resultante desse processo, o organismo tem um sistema de defesa antioxidantes, que conta com a participação da vitamina E (dl- $\alpha$-tocoferol). Quando a LDL se encontra elevada, o colesterol pode se depositar na luz dos vasos sanguíneos, diminuindo o seu calibre e podendo, desse modo, causar o enrijecimento da parede do vaso sanguíneo e a formação de placas de ateroma nos cavalos. Normalmente, os valores desse colesterol LDL são obtidos pela equação de Friedewald, por isso neste estudo objetivou-se determinar os valores de colesterol LDL por espectrofotometria em equinos submetidos à exercício físico em esteira e suplementados com vitamina "E". Para tal, foram selecionados dez cavalos hígidos da raça Árabe e Crioulo, em que as amostras foram processadas em duplicata utilizando os kits comerciais LDL Direto (BioClin ${ }^{\circledR)}$ por meio de espectrofotômetro semiautomático BA-88A (Mindray®). Neste estudo, não se obteve diferença estatística significante entre os tempos analisados e nem com a suplementação de vitamina "E", com o protocolo estabelecido.
\end{abstract}

Palavras-chave:cavalos, lipídios, espectrofotômetro, antioxidante.

\section{DETERMINATION OF EQUINE'S LDL CHOLESTEROL BY SPECTROPHOTOMETRY SUBMITTED TO EXERCISE ON TREADMILL AND SUPPLEMENTED WITHVITAMIN "E"}

\begin{abstract}
Low density lipoprotein (LDL) is part of a group of lipoproteins which in amphiphilic nature, are responsible for transporting cholesterol plasma, it is important entraiment, since cholesterol is insoluble in blood plasma. It is known that the main power source of the horses to the basal contraction of the muscle, is obtained from the free fatty acids from adipose tissue

\footnotetext{
${ }^{1}$ Graduanda em Medicina, Universidade Vila Velha (UVV)

${ }^{2}$ Centro Ciências Agroveterinária UDESC - Av. Luis de Camões 2090- Conta Dinheiro, Lages - SC, 88520-000.

${ }^{3}$ Depto. Cirurgia e Anestesiologia Veterinária - FMVZ-UNESP

${ }^{4}$ Depto. Clínica Veterinária, FMVZ-UNESP

${ }^{5}$ Pós-graduanda em Ciência Animal, Universidade Vila Velha - UVV
}

Costa PF, Oliveira, MCG, Yonezawa LA, Watanabe MJ, Kohayagawa A, Barbosa TS. Determinação dos valores de colesterol-LDL de equinos por espectrofotometria submetidos a exercício em esteira e suplementados com vitamina "E". Vet. e Zootec. 2018 mar.; 25(1): 112-119.
\end{abstract}


and ketone bodies, breakage products of fatty acids from the liver, a process that generates species reactive oxygen (ROS), which, in excess, leads to cellular oxidative stress. To avoid the resulting damage of this process, the body has an antioxidant defense system, which includes the participation of vitamin E (dl- $\alpha$-tocopherol). When LDL is high, cholesterol can be deposited in the lumen of blood vessels, reducing their size and can thus cause the hardening of the blood vessel wall and the formation of atheroma in horses. Normally, the values that LDL cholesterol are obtained by the Friedewald equation, so this study aimed to determine the LDL cholesterol levels by spectrophotometry in horses submitted to exercise on a treadmill and supplemented with vitamin "E". To this ten healthy Arabe and Criolo horses were selected. The samples were processed in duplicate using the Direct LDL commercial kits $\left(\right.$ BioClin $^{\circledR}$ ) by means of semi-automatic spectrophotometer BA-88A (Mindray ${ }^{\circledR}$ ). This study did not present a statistically significant difference between the analyzed time and not with vitamin supplementation "E" with the established protocol.

Keywords: horses, lipids, spectrophotometer, antioxidants

\title{
DETERMINACIÓN DE LOS VALORES DE COLESTEROL LDL EQUINA POR ESPECTROFOTOMÉTRICA SOMETIDOS A EJERCICIO EN ESTERA Y SUPLEMENTADOS CON VITAMINA "E"
}

\begin{abstract}
RESUMÉN
La lipoproteína de baja densidad (LDL) es parte de un grupo de lipoproteínas que en la naturaleza anfifílica, son responsables de transportar el colesterol en plasma, siendo el arratre importante, ya que el colesterol es insoluble en el plasma sanguíneo. Se sabe que la fuente de alimentación principal de los caballos a la contracción basal del músculo, se obtiene a partir de los ácidos grasos libres del tejido adiposo y los cuerpos cetónicos, productos de rotura de los ácidos grasos del hígado, un proceso que genera especies reactivas de oxígeno (ROS), que, en exceso, conduce a estrés oxidativo celular. Para evitar el daño resultante de este proceso, el cuerpo tiene un sistema de defensa antioxidante, que incluye la participación de la vitamina E (DL- $\alpha$-tocoferol). Cuando LDL es alta, el colesterol puede ser depositado en el lumen de los vasos sanguíneos, lo que reduce su tamaño y por lo tanto puede causar el endurecimiento de la pared del vaso sanguíneo y la formación de placas de ateroma en los caballos. Normalmente, los valores de colesterol LDL que se obtienen mediante la ecuación de Friedewald, por lo que este estudio tuvo como objetivo determinar los niveles de colesterol LDL por espectrofotometría en caballos sometidos a ejercicio en estera y suplementados con vitamina "E". Para esto diez caballos sanos fueron seleccionados de la raza Criollo y Àrabe, en el que las muestras se procesaron por duplicado utilizando los kits comerciales de LDL directa $\left(\right.$ BioClin $\left.^{\circledR}\right)$ por medio de espectrofotómetro semi-automática BA-88A (Mindray ${ }^{\circledR}$ ). Este estudio no presentó una diferencia estadísticamente significativa entre el tiempo analizado y no con la administración de suplementos de vitamina "E" con el protocolo establecido.
\end{abstract}

Palabras clave: cavallos, lípidos, espectrofotómetro, antioxidante.

\section{INTRODUÇÃO}

Os lipídeos são a segunda maior fonte de energia em equinos, sendo no exercício intenso considerados a principal fonte (1). Para tanto, eles são digeridos no intestino delgado sobre ação das lipases e, concomitantemente, emulsificados pelos sais biliares $(2,3)$. 
Finalmente, os ácidos graxos formados no processo são absorvidos no íleo distal, ceco ou intestino grosso(3). Sendo que, é no intestino grosso que há uma grande digestão, mediada pela microbiota local, que produz ácidos graxos voláteis essenciais no metabolismo energético (4). A produção de energia a partir dos lipídeos é feita pelo processo de oxidação aeróbica, em que o ácido graxo é oxidado e se torna energia pronta para o uso sob a forma de adenosina trifosfato (ATP) (5).

Dentre os lipídeos, o colesterol é um dos mais relevantes no organismo, que se apresenta no sangue ligado à lipoproteína, como a lipoproteína de baixa densidade (LDL), conhecido como "mau colesterol" (6). A LDL apresenta a função de transportar lipídeos do fígado para os tecidose, em excesso, é altamente aterogênica(7).Nos cavalos, a LDL é responsável pelo transporte de $15 \%$ do colesterol plasmático, apesar de ser a lipoproteína com maior concentração de colesterol, tendo como principal função fornecer colesterol para os tecidos periféricos(8). Seu peso em equinos é composto $6 \%$ por triacilglicerol, $8 \%$ por colesterol, $36 \%$ por ésteres, $23 \%$ por fosfolipídeos e $27 \%$ por proteína (apolipoproteína)(9).

Usualmente, o valor da LDL é estimado indiretamente a partir do valor do colesterol e duas de suas frações, que são lipoproteína de alta densidade (HDL) e lipoproteína de muito baixa densidade (VLDL) aplicados na equação de Friedewald[Colesterol LDL = Colesterol total - (colesterol-HDL + triglicerídios/5)], em seres vivos com predomínio da LDL no organismo $(7,10)$. Porém, nos equinos, há o predomínio do colesterol-HDL, o qual se revela aumentado em resposta a maior ingestão de gordura e de colesterol, logo o uso da equação para cálculo da concentração sérica de LDL poderia enviesar os valores da $\operatorname{LDL}(10,11)$. Ademais, o método não é apropriado para determinar perfil lipídico sob condições de dislipidemias, como visto em humanos (12).

Em equinos submetidos a exercício físico exaustivo, esses lipídeos sofrerão maior lipoperoxidação, pois, com a atividade física, há o aumento acentuado da respiração celular com maior síntese de radicais livres, responsáveis pelo dano oxidativo que afeta a integridade celular e desencadeiam tal processo de oxidação lipídica $(13,14)$. Portanto, para evitar os efeitos danosos do estresse oxidativo à célula, o organismo conta com um sistema antioxidante, no qual um de seus componentes é a vitamina $\mathrm{E}$ (dl- $\alpha$-tocoferol)(14,15). Logo a suplementação de equinos com vitamina $\mathrm{E}$ reduz a produção de radicais livres, pois essa vitamina converte os radicais livres em espécies mais estáveis e, portanto, menos reativas(16). Essa ação antioxidante do tocoferol é verificada laboratorialmente por uma diminuição de malondialdeído, que é um marcador de injúria celular (17).

Assim, devido à importância dos lipídios no metabolismo equino e à não padronização dos valores basais de LDL usando a mensuração direta pelo espectofotômetro, objetivou-se no presente estudo determinar os valores de colesterol-LDL por espectrofotometria e verificar a influência da vitamina "E” nos níveis séricos dessa lipoproteína.

\section{MATERIAL E MÉTODOS}

\section{Animais}

O trabalho foi realizado sob a aprovação do comitê de ética com o protocolo $\mathrm{n}^{\circ} 128 / 2009$ CEUA/ UNESP - Botucatu.

Utilizou-se 10 equinos, sendo cinco da raça Puro Sangue Árabe e cinco da raça Crioula, mantidos em piquetes do Hospital Veterinário da Faculdade de Medicina Veterinária e Zootecnia (FMVZ) da Universidade Estadual Paulista (Unesp), Campus de Botucatu, sob as mesmas condições de manejo alimentar e sanitário. Os animais estavam sem treinamento físico por mais de um ano, com idade variando de oito a dez anos, e peso médio de $372,1 \pm$ $32,9 \mathrm{~kg}$, foram considerados hígidos mediante exames hematológico e bioquímico sérico. 


\section{Delineamento experimental}

Os equinos foram submetidos a um teste padrão de exercício progressivo em esteira de alta velocidade (Mustang 2200 AG, Kagra, Suíça) inclinada a 6\%, da qual a velocidade é elevada gradualmente, com o protocolo de exercício proposto por Watanabe et al. (18). Utilizou-se a máscara de análise de trocas gasosas e dados ventilatórios (Metavet, Cortex, Alemanha), para se extrair a carga de trabalho para cada equino, com base no consumo máximo de oxigênio $\left(\mathrm{VO}_{2} \operatorname{máx}\right)(19)$, sendo o valor médio dos animais de 110,4 $\pm 20,4$ $\mathrm{mL} / \mathrm{kg} / \mathrm{min}$.

Após 14 dias do teste progressivo, os equinos foram submetidos ao teste de baixa intensidade e longa duração (TLD1). O teste, considerado de exercício predominantemente aeróbico (concentração de lactato inferior a $4 \mathrm{mmol} / \mathrm{L}$ ), foi realizado com a esteira inclinada a $6 \%$, à velocidade de $35 \%$ do $\mathrm{VO}_{2}$ máx de cada animal pelo período de $60 \mathrm{~min}$ (20), correspondendo a uma velocidade média de $2,3 \pm 0,5 \mathrm{~m} / \mathrm{s}$.

A suplementação da vitamina $\mathrm{E}$ (dl- $\alpha$-tocoferol) dos animais teve início logo após o teste TLD1, na dose de 1.000 UI/animal (21), por meio de cápsulas gelatinosas (E-tabs 1000 UI, Sigma Pharma, Hortolândia, Brasil) misturadas a raspas de rapadura, por via oral, diariamente e sem interrupção até o final do experimento. Após 59 dias do início da suplementação, os equinos realizaram o segundo teste (TLD2) com o mesmo protocolo de TLD1. A temperatura e a umidade relativa do ar do salão do Centro de Medicina Esportiva Equina da Unesp de Botucatu, onde os testes foram realizados, variou de 17,0 a 20,6 $6^{\circ} \mathrm{C}$ e 66 a $81 \%$, respectivamente.

\section{Colheita e processamento das amostras}

As coletas de sangue foram realizadas antes do teste (M0), imediatamente após o exercício (PE) e 12h subsequente, sendo armazenadas em tubos contendo ativador da coagulação para obtenção de soro e foram imediatamente separados e congelados a $-80^{\circ} \mathrm{C}$ até serem processadas.

Foi então determinada a concentração dos triglicerídeos séricos pelo método enzimático com kit comercial Bioclin ${ }^{\circledR}$ em analisador bioquímico semiautomático Mindray ${ }^{\circledR}$, modelo BA-88A, no laboratório de Patologia Clínica da Universidade Vila Velha.

\section{RESULTADOSE DISCUSSÃO}

Antes do exercício (M0), as dosagens de LDL-colesterol na ausência de suplementação com vitamina E obteve-se umamédia de $24,11 \pm 7,37 \mathrm{mg} / \mathrm{dL}$. Já para as amostras de LDLcolesterol de equinos com suplementação com vitamina E, obteve-se média de $30,78 \pm 20,74$ $\mathrm{mg} / \mathrm{dL}$.

Logo após o exercício (PE), as dosagens de LDL-colesterol na ausência de

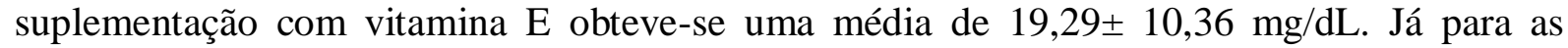
amostras de LDL-colesterol de equinos com suplementação com vitamina E, obteve-se média de $26,11 \mathrm{mg} / \mathrm{dL} \pm 10,68 \mathrm{mg} / \mathrm{dL}$.

Já no momento 12 horas após o exercício $(12 \mathrm{~h})$, as dosagens de LDL-colesterol na ausência de suplementação com vitamina E obteve-se uma média de 12,65 \pm 7,53 mg/dL. Já para as amostras de LDL-colesterol de equinos com suplementação com vitamina E, obtevese média de $26,11 \mathrm{mg} / \mathrm{dL} \pm 10,68 \mathrm{mg} / \mathrm{dL}$. 
Todos os resultados estão apresentados na figura 1, sendo em TDL1 os equinos ainda sem suplementação e TDL2 o momento com suplementação de vitamina D.

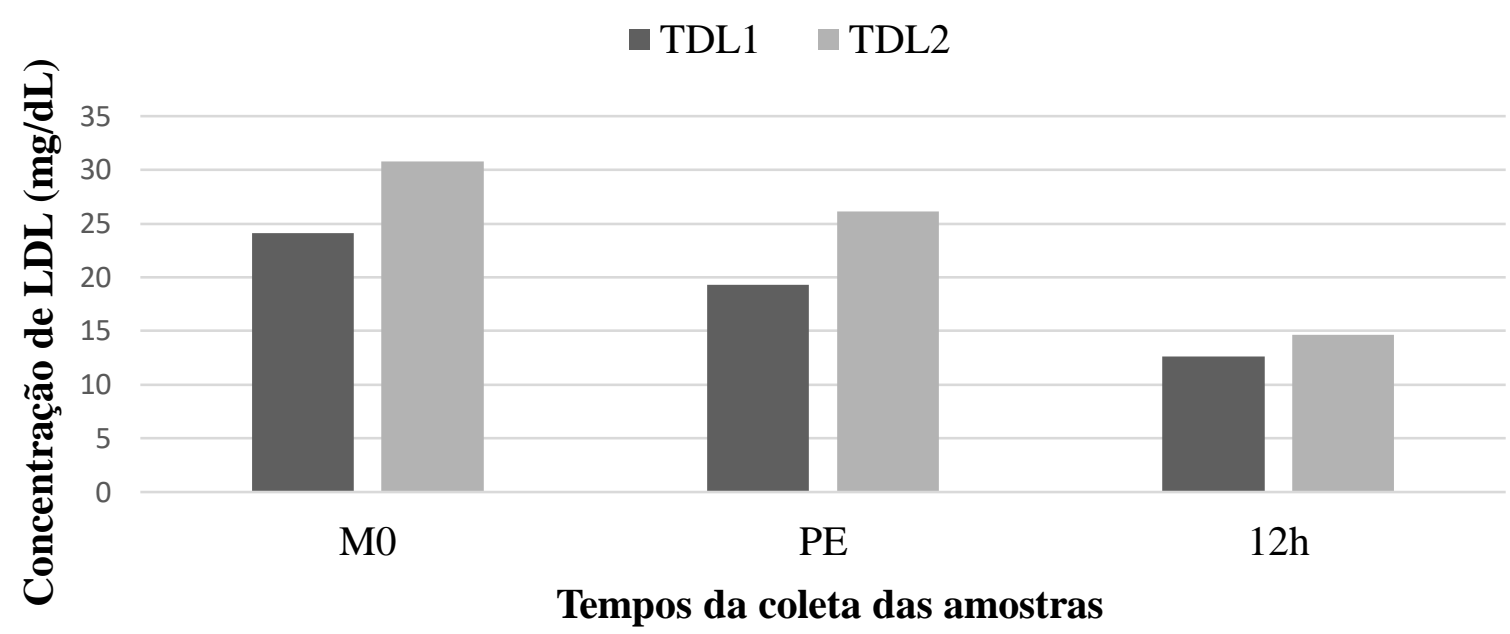

M0 = LDL no repouso; PE = LDL imediatamente após o exercício; 12 h = LDL em horas após o exercício.

Figura 1. Comparação da dosagem de LDL em equinos com e sem a suplementação de vitamina E.

O estudo de Ozório e Velázquez (10), realizado com 200 cavalos revelou com o método de precipitação média de $32 \mathrm{mg} / \mathrm{dL}$, com desvio padrão de $8,8 \mathrm{mg} / \mathrm{dL}$, sendo que o valor mínimo foi de $13 \mathrm{mg} / \mathrm{dL}$ e o valor máximo de $59 \mathrm{mg} / \mathrm{dL}$. No mesmo estudo, determinada a dosagem de LDL pelo método de Friedewald, encontrou-se média de $29 \mathrm{mg} / \mathrm{dL}$, com desvio padrão de 7,0 mg/dL e valores mínimos e máximos de 11,2 e $54,2 \mathrm{mg} / \mathrm{dL}$, respectivamente (10). Comparando-se tais resultados com os do presente estudo, observou-se que os valores mínimo e máximo obtidos por espectrofotometria se encontram dentro dos valores mínimos e máximos obtidos tanto por precipitação quanto pela fórmula de Friedewald, possivelmente pela semelhança na amostra, ou seja, cavalos de idade semelhante, mantidos adequadamente e com boas condições de saúde. Além disso, outra pesquisa realizada pelos mesmos autores (22), também feita pelo método direto e realizada com 200 equinos, encontrou valor médio de LDL-colesterol correspondente a $27,9 \mathrm{mg} / \mathrm{dL}$, valor mínimo de $14,0 \mathrm{mg} / \mathrm{dL}$ e valor máximo de $51 \mathrm{mg} / \mathrm{dL}$.Os valores obtidos são similares aos apresentados nesse estudo, possivelmente pela determinação da dosagem direta da LDL nos dois estudos.

Assenza et al. (23) em seu estudo realizado a partir da análise de 34 cavalos em programa de treinamento obteve, no dia zero do estudo, média do LDL-colesterol correspondente a $72,15 \mathrm{mg} / \mathrm{dL}$, com desvio-padrão de $14,68 \mathrm{mg} / \mathrm{dL}$ para cavalos de trote e média de 45,26 mg/dL, com desvio-padrão de $21,13 \mathrm{mg} / \mathrm{dL}$ para cavalos puro sangue. Ambas as médias diferem das encontradas no presente estudo, possivelmente por se tratar de condições esportivas e raças diferentes de cavalos, que são de trote e de puro sangue.

Ao utilizar a fórmula de Friedewald em 92 cavalos de corrida da raça árabe e mestiça encontrou-se média de $35 \mathrm{mg} / \mathrm{dL}$ de LDL-colesterol, com desvio-padrão de $1,54 \mathrm{mg} / \mathrm{dl}$, em estudo de Hasso et al. (24) ${ }^{10}$. Tal resultado se encontra dentro dos valores obtidos no presente estudo, possivelmente pela semelhança das raças, uma vez que ambos utilizam cavalos árabes. Porém, a diferença entre as médias pode se dar pela utilização da fórmula de Friedewald, diferindo do estudo em questão que usou o método de espectrofotometria.

Já no estudo de Gonzaga at al (25), usando seis cavalos suplementados com um antioxidante gama-orizanol, foram obtidos uma média e um desvio padrão de LDL-colesterol, 
calculado pela fórmula de Friedewald, de $38,44 \mathrm{mg} / \mathrm{dL}$ e $10,38 \mathrm{mg} / \mathrm{dL}$, respectivamente. Essa substância reduz o colesterol plasmático, diminui a biossíntese do colesterol hepático, reduz a absorção do colesterol e aumenta a excreção de ácidos biliares nas fezes (25). Essa diferença encontrada nos estudos pode ser explicada, possivelmente pela utilização de diferentes tipos de antioxidantes.

Com o passar do tempo de treinamento foi observado um aumento dos níveis de LDL, fato observado pelas médias de TDL2 que foram superiores a TDL1. Tal comportamento foi observado também por $\operatorname{Kurcz}(21)$, provavelmente, porque o exercício aumenta a utilização de lipídeos da dieta e sua mobilização como fonte de energia. Entretanto os resultados encontrados no presente estudo não apresentaram diferença estatisticamente significante.

A redução dos níveis plasmáticos de LDL após o exercício pode ser explicada pelo reflexo da redução do colesterol plasmático, que especialmente em esforços, é utilizado para síntese de cortisol pela glândula adrenal e pela sua utilização no metabolismo aeróbico(1). Porém as reduções dos valores da LDL encontrada após o exercício não apresentaram diferença estatisticamente significante.

\section{CONCLUSÃO}

O presente estudo apresenta valores de LDL-colesterol por espectrofotometria em equinos, visando contribuir valores de referência basais por meio da dosagem direta. Entretanto, ainda há necessidade de estudos para a determinação de valores de referência de LDL-colesterol. A partir do estudo não é possível afirmar que a vitamina $\mathrm{E}$ altera os níveis séricos da LDL, pois os dados encontrados não apresentam significância estatística. Assim ainda se sugere mais estudos para determinar a real influência da vitamina $\mathrm{E}$ nos níveis séricos de LDL.

\section{APROVAÇÃO DO COMITÊ DE ÉTICA E BIOSSEGURANÇA}

Todos os procedimentos metodológicos envolvendo os animais foram aprovados pela Comissão de Ética no Uso de Animais do Setor de Ciências Biológicas da Universidade Estadual Paulista no dia 29/09/2009 (n 128/2009-CEUA/ UNESP - Botucatu).

\section{APOIO E FINANCIAMENTO}

Fundação de Amparo à Pesquisa do Estado de São Paulo (FAPESP) n08/57366-9; 09/53314-7, Universidade Vila Velha M03-2014PI008.

\section{REFERÊNCIAS}

1. Noleto PG. Perfil bioquímico de equinos submetidos a provas de esforço físico [dissertação]. Universidade Federal de Uberlândia e Faculdade de Medicina Veterinária e Zootecnia; 2012.

2. Morgado E, Galzerano L. Utilização de óleos em dietas para equinos. Revista Eletrônica de Veterinária, 2006; 7.

3. Ribeiro RM. Inclusão de Gordura na Alimentação de Equinos [tese]. Universidade de São Paulo, Faculdade de Medicina Veterinária e Zootecnia e Departamento de Nutrição e Produção Animal;2007. 
4. Fassio PO et al. Uso do Óleo de Canola na Alimentação de Equinos Atletas. In: II Semana de Ciência e Tecnologia do IFMG do campus Bambuí; 2009. Instituto Federal de Minas Gerais; Zootecnia do campus Bambuí; 2009.

5. Duarte PC. Avaliação do metabolismo energético de cavalos em provas de longa distância: Accutrend® Plus versus laboratório [dissertação]. Universidade de Brasília e Faculdade de Agronomia e Medicina Veterinária; 2013.

6. Santos JE, Guimaraes AC, Diament J. Consenso Brasileiro Sobre Dislipidemias Detecção, Avaliação e Tratamento. Arq Bras Endocrinol Metab. São Paulo,1999; 43:287305 .

7. Motta VT. Bioquímica Clínica para o Laboratório - Princípios e Interpretações. Rio de Janeiro:Editora Medbook, $5^{\text {a }}$ Ed; 2009.

8. Brandi RA. Parâmetrossangüíneos de cavalos de póloemAtividade, suplementados com óleo de girassol[dissertação]. Universidade Federal de Lavras; 2004.

9. Kaneko JJ,Harvey JW, Bruss, ML. Clinical Biochemistry ofDomestic Animals. EstadosUnidos: Elsevier, 6 ${ }^{\mathbf{a}}$ edição; 2008.

10. Osorio, J.H. Determinación de los niveles de colesterol LDL en una espécie conpatrón HDL. Rev. investig. vet. Perú, Lima, 2013; 24.

11. Bauer JE. Metabolismo comparado de lípidos y lipoproteínas. Pet's Ciencia, 1997;13: 362-376.

12. Baigent, C, Burbury K, Wheeler D. Premature cardiovascular disease in chronic renal failure. Lancet, 2000; 35(9224):147-52.

13. Avellini, L.; Chiaradia, E.; Gaiti, A. Effect of exercise training, selenium and vitamin E on some free radical scavengers in horses (Eqquscaballus). Comparative Biochemistry and Physiology Part B, 1999; 123.

14. Vieira WSV. Perfil bioquímico e capacidade antioxidante total em cavalos de polo suplementados com selênio e vitamina E[tese]. Instituto de Veterinária e Universidade Federal Rural do Rio de Janeiro, 2011.

15. Dias DCR. Estresse oxidativo na fisiopatogenia de enfermidades de equinos. In: II simpósio alagoano de medicina equina; 2012, Revista Brasileira de Medicina Equina. Suplemento 1, v. 40. 2012.

16. Catania, A. S.; Barros, C. R. de; Ferreira, S. R. G. Vitaminas e minerais com propriedades antioxidantes e risco cardiometabólico: controvérsias e perspectivas. Arq Bras Endocrinol Metab, São Paulo, 2009; 53:550-9.

17. Yonezawa, L.A, et al. Efeito da suplementação com vitamina E sobre os metabolismos oxidativo e cardíaco em equinos submetidos a exercício de alta intensidade. Arq. Bras. Med. Vet. Zootec., 2015; 67:71-79. 
18. Watanabe, M.J.; Silveira, V.F.; Machado, L.P. et al. Aplicação da espirometria durante teste padrão de exercício progressivo em esteira para avaliação da troca gasosa respiratória de equinos da raça Árabe. Arch. Vet. Sci., v.14, p.17-24, 2009.

19. Kaneko, J.J., Harvey J.W., Bruss, M. L. Clinical Biochemistry of Domestic Animals. Elsevier, 2008; 6.

20. Brandi RA. Parâmetros sangüíneos de cavalos de póloem Atividade, suplementados com óleo de girasol [dissertação]. Universidade Federal de Lavras; 2004.

21. Kurcz EV. Lipoprote in profiles of the horse in response to increased dietary fat and exercise [dissertação]. University of Arizona; 1992.

22. Osorio, O.J.; Uribe-Velazquez, L. Comparación de los métodos Directo y de Fried ewald para la determinación de los niveles de colesterol LDL enel equino. Rev. MVZ Cordoba, Córdoba, 2011; 16.

23. Assenza, A, et al. Lipid Utilization Pathways Induced by Early Training in Standardbred Trotters and Thorough breds. Journal of Equine Veterinary Science, 2012; .32:704-710.

24. Hasso, S. A, et al. Serum glucose concentration and lipid profile in racing horses. Iraqi Journal of Veterinary Sciences, 2011; 26:1-3.

25. Gonzaga, I. V. F. et al. Qualidade espermática e perfil plasmático de lipídeos em garanhões suplementados com óleo de arroz semi-refinado com alto teor de gamaorizanol. Brazilian Journal of Veterinary Research and Animal Science. São Paulo, 2012; 49: 215-220.

Recebido em: Aceito em: 